\title{
Design and Implementation of Alcohol Concentration Monitoring System Based on MCU
}

\author{
LU Qingru, a, HUANG Hui, b , CHEN Debin ${ }^{3, c}$, XIN Haiyan $^{4, d}$ \\ ${ }^{1,2,3,4}$ Department of Electronics,Chengxian Institute,Southeast University,Nanjing Jiangsu \\ 210000, China \\ aemail: silver_1120@163.com, bemail: huanghui_1225@163.com, \\ cemail: 906720556@qq.com, 'email:983511889@qq.com
}

Keywords: Alcohol Concentration; Monitor; MCU; Gas Sensor

\begin{abstract}
With the development of science and technology, intelligent products becomes more and more popular. Alcohol concentration monitoring system based on the MCU STC89C52 and gas sensor has many functions such as alarm by sound and light、 LCD display function and so on. The system can set different thresholds according to different environment in order to meet the different environment monitoring. The warning lights can be taken as two levels of alarm respectively("tipsy" and "pissed" )if the value measured has exceeded the threshold, and the value displays. The system according to the design scheme has the advantages of high sensitivity, good working performance, low power consumption, low cost, high price etc., which can be used in traffic detection, fermentation monitoring in wineries and foods factories etc..
\end{abstract}

\section{Introduction}

Sensing technology has gradually become mature with the rapid development of information technology in the 20th century, and it has been widely used in the production of life. The sensor has played a pivotal role in all fields, and high precision, high reliability, miniaturization, low power consumption and intelligent digital has become the direction of its development.

In order to check for drunk driving, police often use a portable breath alcohol detector by detecting driver exhaled gas in order to judge the drinking driving .It can only judge whether a driver is drunk with the use of alcohol breath tester at present, alcohol concentration only can be measured by some blood test. In view of this situation, the design of the alcohol concentration monitoring system can monitor the concentration of alcohol in the environment, besides it can display the results and numerical classification of warning, you can also set the threshold value of alcohol concentration on manual arbitrarily to delineate the safety limits of alcohol concentration on different environment.It also has other functions just as hierarchical alarm etc. .

\section{Design principle and process}

\section{Design of the hardware}

The framework diagram of this alcohol concentration monitoring system is shown in Fig.1. The system is mainly composed of capacitive touch sensor, gas sensor, analog digital converter, MCU, LCD display, keyboard module and sound and light alarm. 


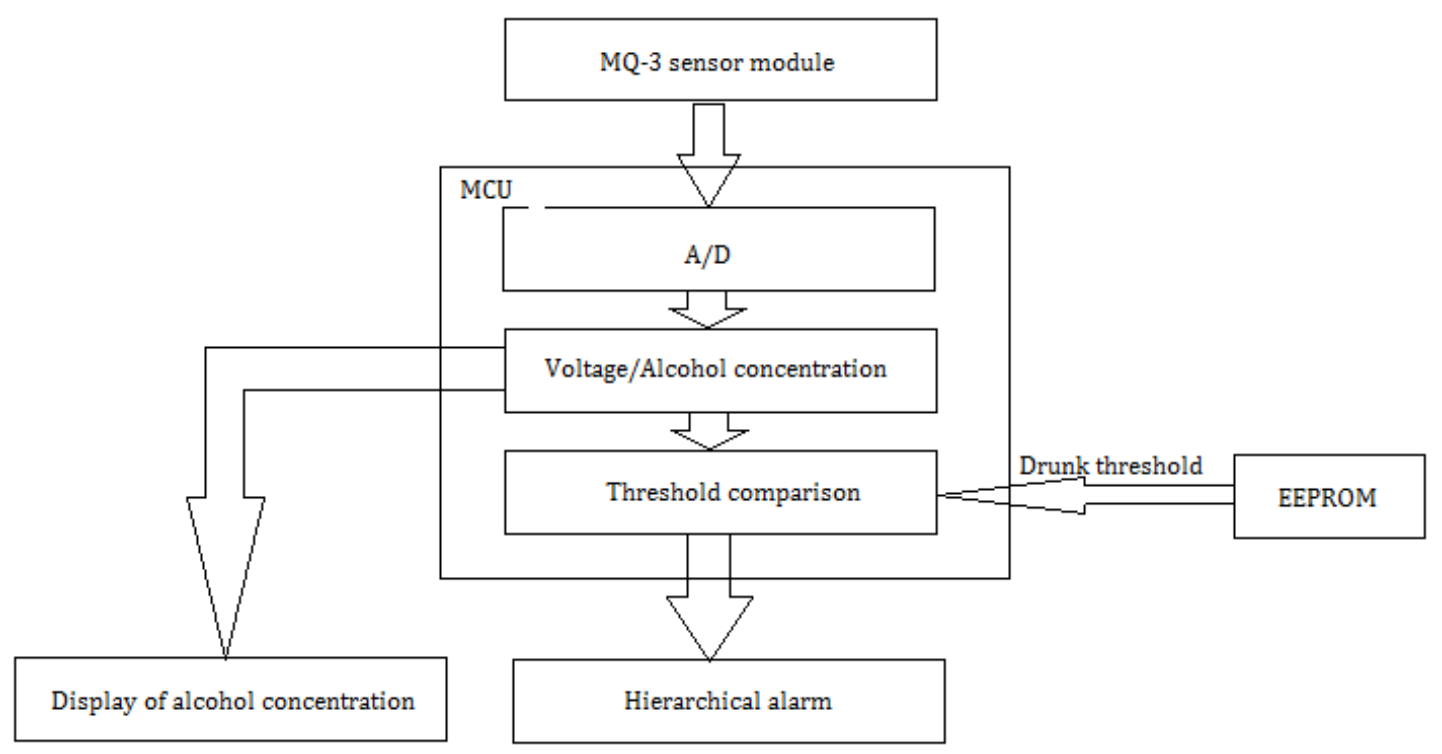

Fig.1. Principle block diagram

Data acquisition is completed by MQ-3 alcohol gas sensor which is an alcohol sensor , alcohol sensor will detect the alcohol concentrations and convert them to electrical signals,the signals are transmitted into an analog-to-digital converter, the converted digital signal is then transmitted into the MCU,it is analyzed and processed by MCU,and the results will be displayed on the screen at last.

Comparing with the threshold of alcohol concentration set by the keyboard, the buzzer will alarm and LED will flash constantly if the alcohol concentration of the environment is detected that the results have exceeded the threshold value,which is controlled by the MUC.

The physical map of MQ-3 alcohol gas sensor module as one of the core devices is shown in Fig.2.The sensitivity of MQ-3 for various gases is different, and it's more sensitive to alcohol than others as shown in Fig.3. Its sensing principle is that the output resistances coming from the gas sensitive resistance change with the concentration of the ethanol gas.

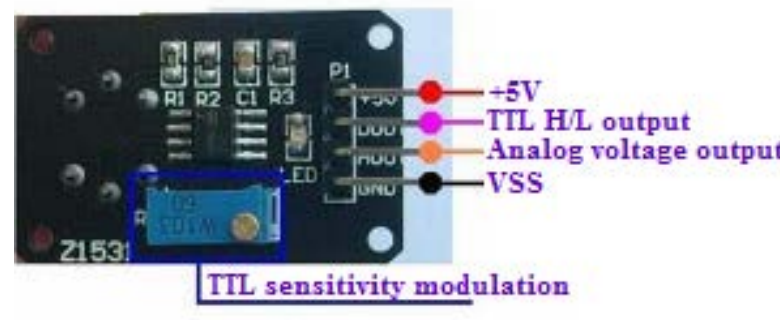

Fig.2. Physical map of MQ-3

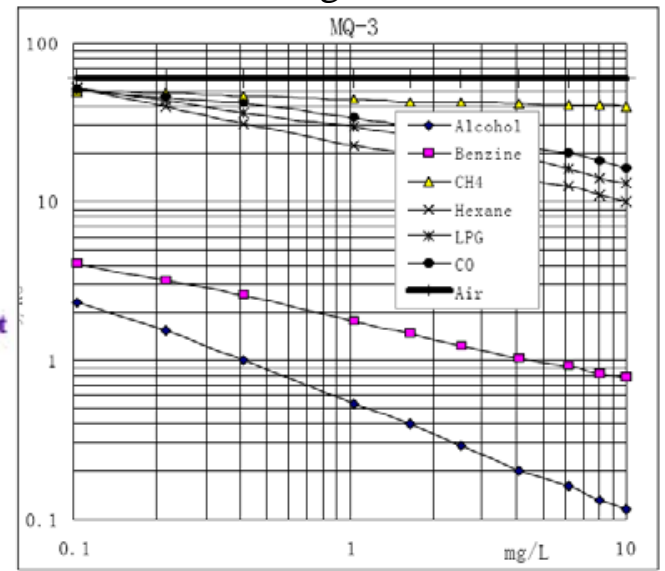

Fig.3. Sensitivity curves for various gases

STC12C5A16AD is chosen as the MCU in this system which is another core module.The chip has 52 cores and 8 bits ,10 multi-channel A/D conversion module is integrated in the internal which is suitable for maintenance circuit in common use. The principle diagram of the MCU system composed of STC12C5A16AD is shown in Figure 4. AOUT is the voltage detection signal which is output by MQ-3 sensor module, and it is transmitted into the port ADC7 then processed there. DOUT is a digital signal output by sensor module, the signal can be output as the alarm signal directly according to the concentration of ethanol gas. The alarm threshold can be adjusted to the potentiometer on the module. 


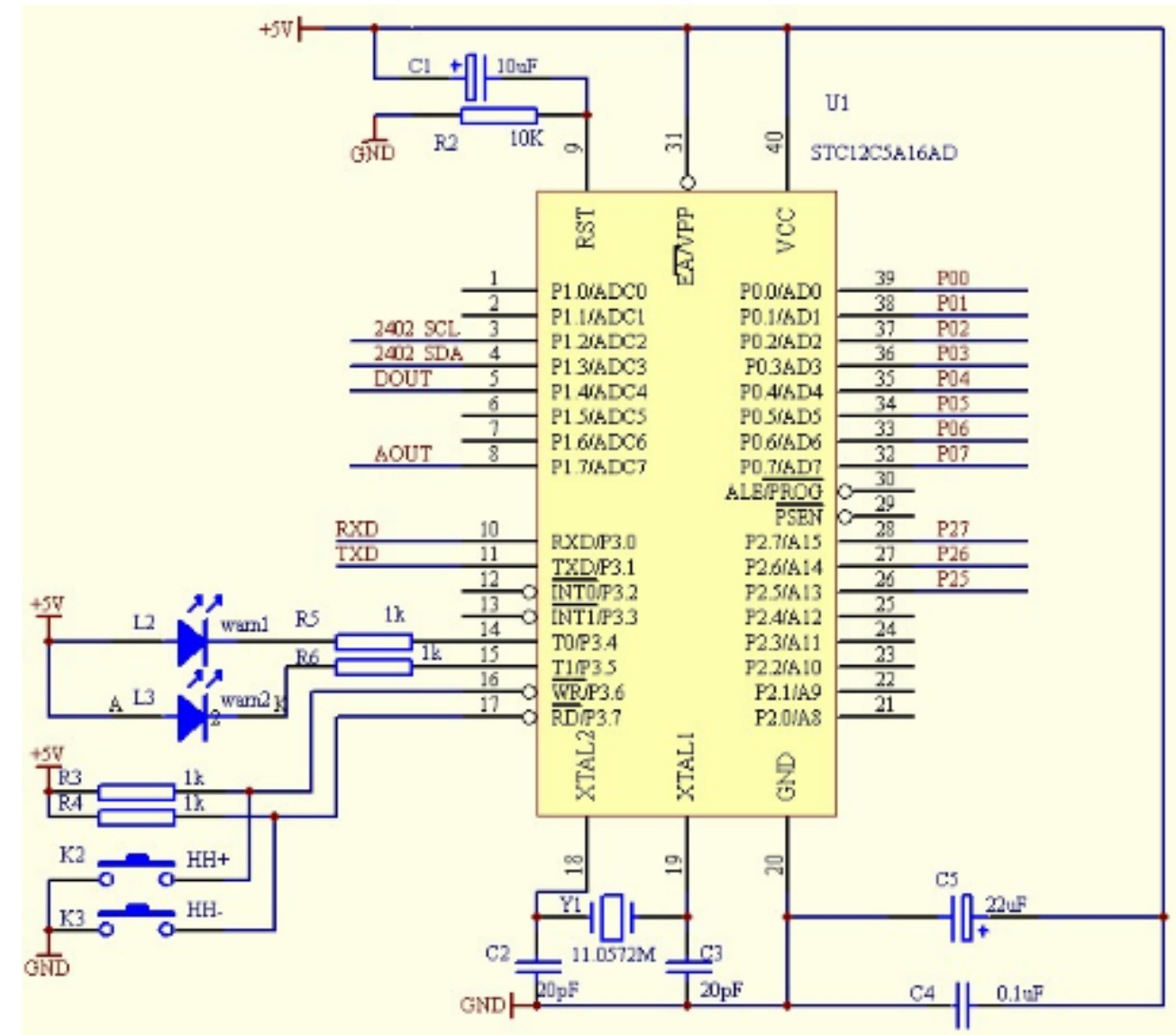

Fig.4. System schematic of STC12C5A16AD

The keys K2 and K3 in Fig.4 are keys as adjustments to the drunken threshold with K2 as "increase" button and K3 as "reduce" button. L2 and L3 are the warning lights which can be taken as two levels of alarm respectively :"tipsy" and "pissed".

SMC1602 liquid crystal screen is used to display the results as the display part in this system. The drunk threshold is stored in the AT24C04 which is an EEPROM chip used for data storage when power is off .

The power of the whole system is supplied with the USB that needs 5V. Programming in the MCU can be handled with the help of chip PL2303 which is in the USB interface.

\section{Design of the software}

The software flow pattern of the system of intelligent alcohol concentration detection is shown in Fig.5. Concentration of alcohol is detected by gas sensor MQ-3 in the environment after the system has been initialized.Processing and judgment to the data conversed by the A/D module is handled by MCU then.It will alarm if the signal from MCU has exceeded the threshold value, and the value of the environment will be shown in the display module meanwhile; the value of the environment will be shown directly if the threshold is not exceeded. 


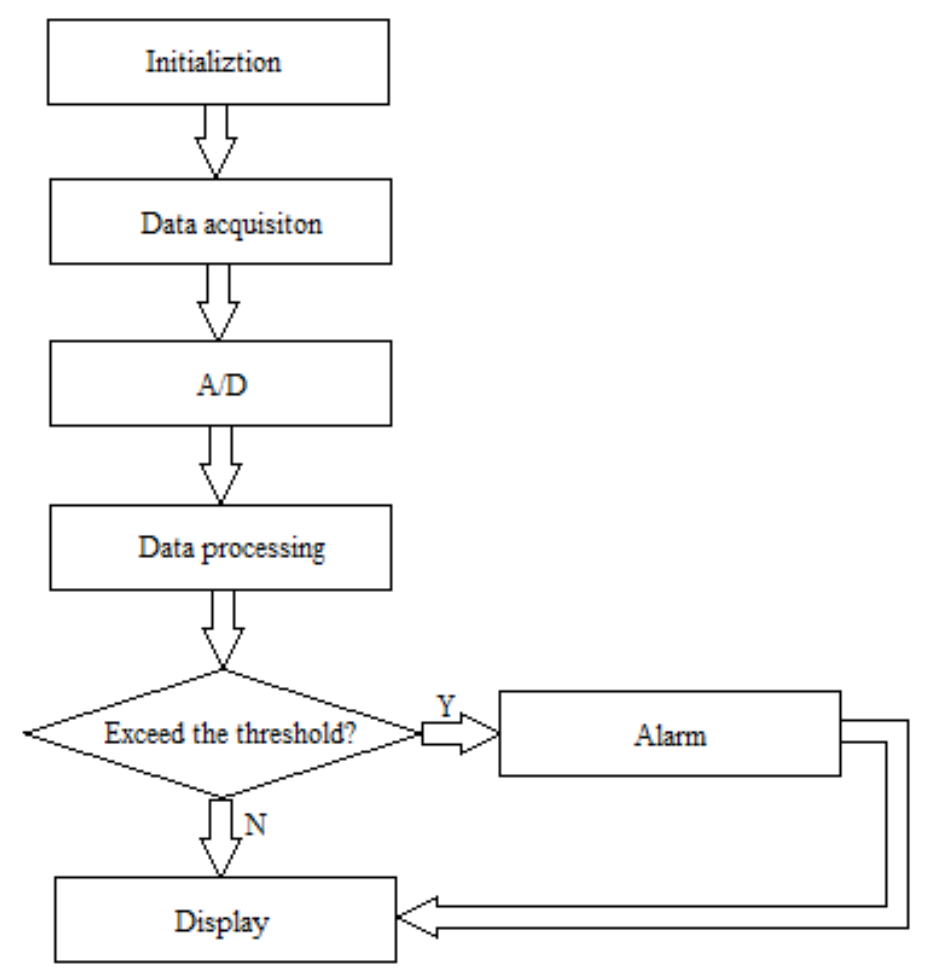

Fig.5. Software flow pattern

\section{Test results}

The final completion of the system according to the design as shown in Fig.6 was test and the results were shown then.

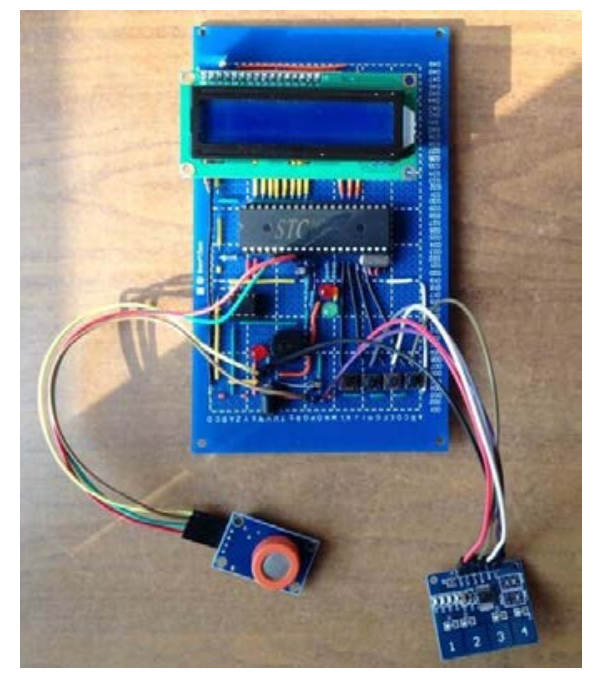

Fig.6. System physical map

The functions of this alcohol concentration monitoring system according to the design can be all realized by text: alcohol concentration threshold setting, sound and light alarm in the different two levels and the display of alcohol concentration.But the response rate of the detector is relatively slow as the result of the lower digits in MCU and the temperature of the environment, temperature has a great influence on the sensitivity of alcohol sensor. On the other hand, the system is very strong anti-interference because alcohol sensor has good stability and selectivity.

\section{Conclusion}

An alcohol concentration monitoring system is designed here. The concentration of alcohol can display in different environment in real time. It can also display grading alarm And in accordance with the threshold which can be set, and so on.The system has the advantages of simple 
structure, small size and convenient carrying, it has high application value and good market prospect.

\section{References}

[1] Zhao Jiagui, New Handbook of Sensor Circuit Design, M., China Metrology Publishing House, 2002:23-26.

[2] Zhang Xin, The Principle and Application of MCU, M.,Electronic Industry Press, 2005:161-258.

[3]Neamen, D.A, Analysis and Design of Electronic Circuit, M.,Electronic Industry Press, 2003:86-104.

[4] Sha Zhanyou, Chinese and Foreign Integrated Sensor Practical Manual, M.,Electronic Industry Press, 2005:200-210.

[5] Huddleston, C. Intelligent Sensor Design:Using the Microchip dsPIC, Posts \& Telecom Press, 2000:827-1124. 